\title{
Avaliação do desempenho da geração de cenários de afluênçias em reservatórios utilizando previsões de precipitação por conjunto
}

\author{
Performance assessment of generation of scenarios for reservoir inflows using \\ ensemble rainfall forecasts
}

\author{
William Cossich ${ }^{1}$, Marcio Cataldi ${ }^{2}$ e Otto Corrêa Rotunno Filho ${ }^{3}$ \\ ${ }^{1}$ Programa de Engenharia Mecânica - COPPE - Universidade Federal do Rio de Janeiro, RJ, Brasil \\ ${ }^{2}$ Departamento de Engenharia Agrícola e Meio Ambiente - Universidade Federal Fluminense, RJ, Brasil \\ ${ }^{3}$ Programa de Engenharia Civil - COPPE - Universidade Federal do Rio de Janeiro, RJ, Brasil
}

\begin{abstract}
Resumo
O planejamento eletroenergético do Sistema Interligado Nacional - SIN tem uma estreita correlação entre os estoques de água dos reservatórios das usinas hidrelétricas e suas afluências futuras. Para o horizonte temporal acima de um mês, são gerados cenários de afluência que não incorporam qualquer tipo de informação climática, e a inclusão dessas informações pode aperfeiçoar a representação das condições hidrológicas futuras. O objetivo deste trabalho é avaliar o ganho que se pode obter com o uso de informações de precipitação na geração de cenários de afluências. Para isso, foram gerados cenários de afluências para a bacia do Rio Grande utilizando a modelagem autoregressiva univariada - AR, que considera apenas afluências passadas, e multivariada - ARx, que considera, além das vazões passadas, informações de precipitação. Para avaliar o desempenho dos cenários gerados usando os dois tipos de modelagem, foram calculados índices estatísticos comparando a vazão prevista com a vazão observada. Os resultados mostraram que ambas as modelagens representaram bem o comportamento sazonal da vazão, no entanto a metodologia univariada capturou, de forma limitada, sua variabilidade natural. Os índices estatísticos evidenciaram o melhor desempenho do modelo $A R x$, indicando que a inserção de informações de precipitação mostra-se como um importante complemento para geração de cenários de afluência.
\end{abstract}

Palavras-chave: Cenários de afluências. Modelos autoregressivos. Previsões de precipitação por conjunto.

\begin{abstract}
The electric energy planning of the National Interconnected System - SIN has a close correlation between present stocks of water in the reservoirs of hydroelectric plants and future inflows. For time periods above one month, inflows scenarios are generated without incorporating any kind of climate information. The inclusion of such information can improve the representation of future hydrological conditions. The objective of this study is to evaluate the gain that can be achieved with the use of precipitation information on generating reservoir inflows scenarios. We generated reservoir inflows scenarios to the Grande River basin using univariate autoregressive modeling - AR, which considers only past inflows, and multivariate modeling - ARx, which also considers precipitation information. Statistical indices were calculated to evaluate the performance of the scenarios generated using the two types of autoregressive models by comparing the predicted inflow with the observed inflow. The results highlighted that both the univariate and multivariate modeling represented well the seasonal behavior of the inflows, however the univariate methodology presented difficulties in capturing the corresponding natural variability. Statistical indices showed the best performance of the ARx model, indicating that the inclusion of the precipitation information proved to be an important addition to generate reservoir inflows scenarios.
\end{abstract}

Keywords: Inflows scenarios. Autoregressive models. Rainfall ensemble forecasts. 


\section{Introdução}

O sistema brasileiro de geração e transmissão de energia elétrica é, em sua maioria, interligado, excetuando-se parte da região norte do país. Esse sistema, denominado Sistema Interligado Nacional - SIN, caracteriza-se por ser hidrotérmico de grande porte, com significativa predominância de geração de origem hidrelétrica.

A geração total de uma determinada fonte de energia varia de acordo com as políticas de geração, que visam otimizar o sistema, a fim de se obter o menor custo da energia para o usuário final, de modo a garantir também o abastecimento a todos os setores da sociedade. No caso da energia hidrelétrica, tem sido observada uma contribuição de cerca de $90 \%$ para o total de geração de energia do país.

Devido a essa característica peculiar, o planejamento e a geração eletroenergética do SIN apresentam uma estreita correlação com os estoques de água existentes nos reservatórios das usinas hidrelétricas e com as afluências a esses reservatórios.

O problema de otimização do planejamento da operação eletroenergética do SIN consiste em minimizar os custos globais de produção de energia elétrica, determinando metas de geração para as usinas hidrelétricas e termoelétricas ao longo de um horizonte de estudo, garantindo o atendimento à demanda de energia elétrica e às restrições operativas do sistema. Para que esse planejamento seja efetivo, permitindo que a modicidade tarifária seja alcançada, deve-se, de forma coordenada, considerar as diferentes dimensões do problema: (i) a dimensão temporal, já que as decisões de armazenamento dos reservatórios das usinas, no tempo presente, influenciam as condições futuras; (ii) a dimensão espacial, pois a disposição e a configuração de bacias hidrográficas do país permitem a presença de usinas em uma mesma cascata; (iii) as incertezas das afluências futuras (PENNA, 2009).

São feitas a previsão e a geração de cenários de afluências aos aproveitamentos hidroelétricos situados nos quatro subsistemas do SIN: sudeste/centro-oeste, sul, nordeste e norte (DAHER DE DEUS, 2008). Essa atribuição, atualmente, pertence ao Operador Nacional do Sistema Elétrico - ONS. No horizonte semanal, as previsões de vazão são obtidas a partir da utilização de modelos estocásticos autoregressivos do tipo vazãovazão, de modelos do tipo chuva-vazão, que podem ser do tipo conceitual-determinístico, com base física, ou de natureza estocástica, incluindo também modelos estocásticos do tipo autoregressivo, ou ainda a partir de técnicas que explorem os fundamentos da inteligência artificial. A partir da segunda semana até um mês a frente, as previsões são feitas a partir da utilização de modelagem autoregressiva do tipo vazão-vazão (GUILHON et al., 2007).

No horizonte temporal acima de um mês, aumentam as incertezas de previsão das afluências, sendo, então, necessária a utilização de séries sintéticas de afluência, obtidas a partir da utilização de modelos estocásticos, usualmente autoregressivos do tipo vazão-vazão (CEPEL, 2002). Esses modelos têm como objetivo gerar séries sintéticas, que, na realidade, são possíveis realizações de um determinando processo estocástico, sob a hipótese de estacionariedade e ergodicidade, premissas comumente adotadas, ainda que sujeitas a questionamentos científicos bastante atuais (CLARKE, 2007). As vazões dessas séries são geradas a partir de modelagem autoregressiva, com a inclusão de ruídos aleatórios, obtidos da série temporal de cada usina e segundo um conjunto de leis probabilísticas. Dessa forma, simulam-se diversas séries temporais que se assemelhem àquelas que poderiam ser geradas pela natureza. Em outras palavras, dada a incerteza associada a esse processo, obtém-se um conjunto de cenários de afluência, que, por sua vez, serão tratados de maneira diferenciada em cada etapa do processo de planejamento da operação do SIN (CEPEL, 2002). No entanto, essa geração de cenários de afluências não incorpora objetivamente qualquer tipo de informação meteorológica ou climática.

Com a evolução da modelagem atmosférica, do conhecimento sobre os fenômenos meteorológicos e climáticos e da ampliação da rede de observações no planeta, a inclusão de informações climáticas pode aperfeiçoar, de maneira significativa, a representação das condições hidrológicas futuras (GODDARD et al., 2010) e, consequentemente, melhorar o planejamento da operação do SIN.

A partir do contexto formulado, o principal objetivo deste trabalho é revelar o ganho que se pode obter com o uso de informações de precipitação na geração de cenários de afluências de vazão, por intermédio da modelagem autoregressiva chuva-vazão em contraste com a abordagem autoregressiva vazão-vazão, no horizonte de até 3 meses.

\section{Modelagem chuva-vazão}

Nas últimas décadas, houve um aumento das pesquisas correlacionando as vazões de bacias hidrográficas com índices e outras variáveis climáticas, além de sua utilização direta no prognóstico de vazões (SOUZA FILHO; LALL, 2003; SANKARASUBRAMANIAN et al., 2008). Além dessa abordagem, previsões de precipitação também têm sido usadas em modelos hidrológicos determinísticos para geração de cenários de vazão (BLOCK et al., 2009). Estudos desse tipo mostram a viabilidade e o ganho que se tem agregando informações climáticas no processo de geração de cenários de afluências.

Guetter e Krauskopf Neto (2006) associaram o erro das previsões hidrológicas às incertezas na estimativa de chuva média sobre a bacia. Por essas razões, a precipitação prevista por modelos numéricos deve, para a maioria dos casos, ser utilizada em associação com outras 
metodologias, como correções estatísticas e adaptações regionais, capazes de minimizar alguns padrões de erros antes dessa informação ser utilizada no modelo hidrológico (HABETS et al., 2004). Uma maneira de se minimizar o erro das previsões, tanto de natureza meteorológica e climática quanto hidrológica, é aplicar a técnica de previsões por conjuntos (ensemble), que surgiu como uma tentativa de aumentar a previsibilidade de modelos dinâmicos atmosféricos. Essa técnica objetiva minimizar as incertezas impostas pelas limitações da previsão numérica da atmosfera, consistindo na passagem de uma única previsão numérica determinística para uma abordagem probabilística (BUIZZA, 2005).

Outra metodologia que pode ser empregada para melhorar as previsões de precipitação são técnicas de correção de viés, que consistem na remoção de possíveis erros sistemáticos que os modelos apresentam em seus resultados (SANKARASUBRAMANIAN et al., 2008; BRAGA et al., 2009).

Block et al. (2009) destacaram a importância de se considerar metodologias que acoplem diferentes Modelos de Circulação Geral da Atmosfera - MCGA com modelos hidrológicos para previsão de vazões, tendo, assim, diferentes membros de uma previsão por conjuntos, de maneira que se torne possível contabilizar a incerteza cumulativa de diferentes fontes, como as condições iniciais, a estrutura dos modelos e os processos modelados.

A previsão de precipitação também pode ser empregada a modelos estocásticos para gerar cenários de afluências. Sankarasubramanian et al. (2008) empregaram um método de regressão de componentes principais para previsão sazonal de vazão utilizando previsões de precipitação provenientes do modelo ECHAM 4.5. Os autores concluíram que é viável a aplicação da remoção de viés, por intermédio da técnica de análise de componentes principais, às previsões de precipitação geradas pelo modelo.

Com os resultados dos trabalhos citados, observa-se que a previsão de vazão, utilizando as técnicas e variáveis apresentadas, pode, de alguma forma, melhorar a otimização do planejamento do SIN. Alguns exemplos de projetos que têm aplicado as previsões climáticas na cadeia do processo de tomada de decisões, no que tange ao planejamento e operação de reservatórios de usinas hidrelétricas em diferentes regiões do planeta, também são apresentados no trabalho de Goddard et al. (2010).

\section{Materiais e Métodos}

Para a elaboração deste estudo, foram utilizadas as previsões climáticas do modelo ECHAM 4.5 aplicadas a um modelo autoregressivo do tipo ARx. Os resultados das previsões obtidas por esse modelo foram comparados com os obtidos por um modelo também autoregressivo do tipo AR, porém sem a inclusão de informações de previsões climáticas.

\section{1 Área de estudo}

A região de estudo deste trabalho abrangeu o trecho da bacia do rio Grande a montante da UHE Furnas. Esse trecho da bacia corresponde à área delimitada pelas regiões 1 a 5 na Figura 1. Possui cerca de 52.138 km², estando localizada no sul do estado de Minas Gerais, entre $20,3^{\circ} \mathrm{S}$ e $22,9^{\circ} \mathrm{S}$ de latitude e $43,6^{\circ} \mathrm{O}$ e $46,8^{\circ} \mathrm{O}$ de longitude.

Segundo o ONS, o subsistema SE/CO, onde está inserida a bacia do rio Grande, produziu $46,5 \%$ da energia hidrelétrica gerada no Brasil no ano de 2010. O valor percentual representa a importância desse subsistema para geração de energia do país, colocando-o como o principal subsistema do SIN. A bacia que mais gerou energia, nesse subsistema, no ano de 2010, foi a bacia do rio Grande. Na usina hidrelétrica de Furnas, está situado um dos maiores reservatórios de regulação do subsistema Sudeste, sendo, portanto, o planejamento da sua operação de grande importância para operação de todo o SIN

Além da importância da bacia selecionada, também é preciso considerar a disponibilidade de dados de vazão e precipitação da região de estudo.

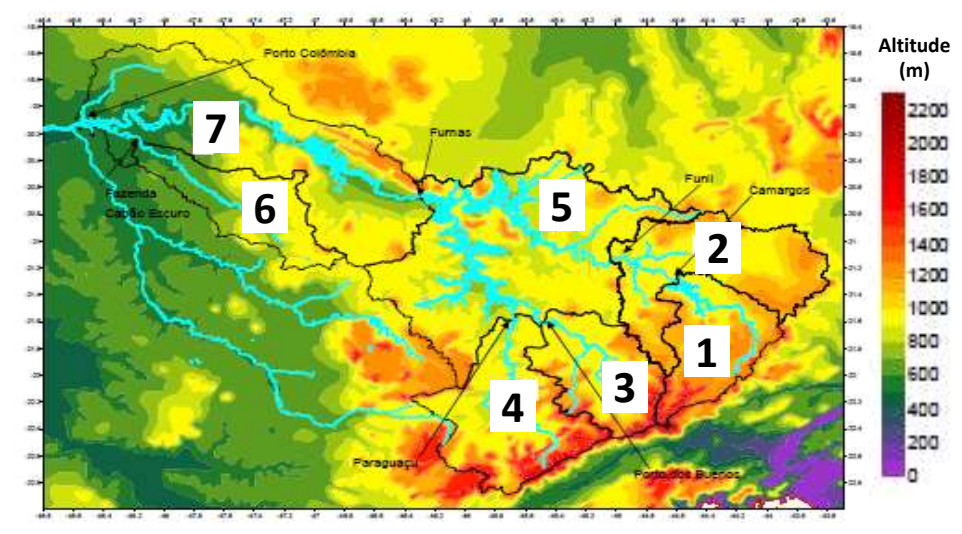

Figura 1 - Distribuição geográfica das sub-bacias da bacia do rio Grande; as regiões 1 a 5 correspondem ao trecho a montante da UHE Furnas. Fonte: adaptado de Saturnino Braga et al., 2009 


\subsection{Previsões Climáticas}

As previsões climáticas obtidas por MCGA's vêm sendo avaliadas de forma contínua e criteriosa pela comunidade científica de todo o globo. Cataldi et al. (2012) analisaram os resultados da previsão de precipitação de diferentes MCGA's para as bacias do rio Grande, no trecho a montante da UHE Furnas, e do rio Paranapanema, situada entre os estados do Paraná e São Paulo. Os modelos estudados foram: ECHAM 4.5 - desenvolvido pelo Max Planck Institute (MPI); CFS - desenvolvido pelo National Center for Environmental Prediction (NCEP); COLA/IRI - desenvolvido pelo antigo NMC, atual NCEP; e o CCM3 - desenvolvido pelo National Center for Atmospheric Research (NCAR).

A partir de dados históricos dos modelos, foi possível perceber que os erros absolutos das previsões de precipitação eram muito altos, sendo necessário aplicar técnicas de correção de viés a essas previsões. Os autores utilizaram duas metodologias: i) ajuste linear; ii) ajuste baseado numa função densidade de probabilidade. Os autores observaram, nesse estudo, que o ajuste linear das previsões é uma técnica mais fácil de ser aplicada, além de ser mais efetiva para correção de erros sistemáticos encontrados nas previsões de precipitação. Foi notado ainda que, após aplicar a técnica de remoção de viés por ajuste linear, as previsões de precipitação do modelo ECHAM 4.5 apresentou um melhor desempenho para a bacia do rio Grande.

Como mencionado previamente, o modelo ECHAM foi desenvolvido pelo Instituto Max Planck de Meteorologia - MPI. Uma detalhada descrição desse modelo pode ser encontrada em Roeckner et al. (1996).

Para o desenvolvimento do presente trabalho, foi utilizada a sua versão 4.5 , baseada no modelo de previsão de tempo do Centro Europeu de Previsão de Tempo de Médio Alcance -ECMWF, que sofreu diversas modificações, tanto numéricas quanto físicas, em relação às suas versões anteriores (ROECKNER et al., 1996).

Nessa versão, o modelo apresenta 19 níveis verticais híbridos, usados em um sistema sigma de coordenadas de pressão. Esse domínio vertical estende-se desde a superfície até o nível de pressão de $10 \mathrm{hPa}$, sendo que a região da camada limite atmosférica apresenta a maior resolução vertical. Exceto para componentes de água, os prognósticos das variáveis são representados por harmônicos esféricos com truncamento triangular no número de onda 42 - T42, apresentando um espaçamento horizontal médio entre os pontos de grade de aproximadamente $2,8 \mathrm{~km}$.

No presente trabalho, foi adotada a metodologia de correção de viés da precipitação de Cataldi et al. (2012), baseada no uso de um fator de correção linear para a precipitação prevista.

Para obter esse fator de correção, foi tomada, inicialmente, a climatologia do modelo ECHAM 4.5 para o período de 1971 a 2000. Em seguida, essa climatologia foi comparada aos dados observados no mesmo período, considerando a média de 22 postos pluviométricos de observação obtidos junto a Agência Nacional de Águas - ANA.

As previsões do modelo ECHAM 4.5 utilizadas neste estudo foram obtidas junto ao endereço eletrônico do International Research Institute for Climate and Society - IRI, utilizando, como condições de contorno, as anomalias da temperatura da superfície do mar - TSM persistidas. O período de testes da geração de cenários de afluências de vazão correspondeu ao período de janeiro de 2002 a dezembro de 2007. Assim, foram obtidas as previsões por conjunto do modelo ECHAM 4.5, considerando vinte e quatro membros de previsão, obtidos a partir de 24 condições iniciais diferentes, até novembro de 2007, válidas para o horizonte de um a três meses. Dessa maneira, foram consideradas as previsões feitas, com um a três meses de antecedência, para todos os meses do período de testes.

\subsection{Modelagem Autoregressiva}

Para geração dos cenários de vazão, foram adotados, neste estudo, os valores de precipitação previstos com remoção de viés, bem como os dados de precipitação observados e a vazão natural observada afluente à UHE Furnas.

Para construção dos modelos autoregressivos simples - AR, foram utilizados somente os dados de vazão observada, enquanto, para elaboração do modelo autoregressivo multivariado - ARx, considerou-se, além da vazão observada, a precipitação observada, bem como um fator que variou de um a doze, de acordo com o mês dos demais dados observados, para representar a sazonalidade no modelo. Ambos os tipos de modelos foram implementados utilizando o programa computacional Matlab®.

Modelos autoregressivos descrevem como uma observação depende diretamente de uma ou mais observações feitas em períodos anteriores. Eles estimam a variável dependente a partir de valores assumidos por ela em tempos anteriores, o que significa dizer que a própria variável é capaz de explicar a sua ocorrência partindo dos dados registrados na série temporal (Wilks, 2006).

A equação geral para um modelo AR de ordem $p-$ $\mathrm{AR}(\mathrm{p})$ é dada pela Equação 1.

$$
x_{t}-\bar{x}=\phi_{1}\left(x_{t-1}-\bar{x}\right)+\phi_{2}\left(x_{t-2}-\bar{x}\right)+\ldots+\phi_{p}\left(x_{t-p}-\bar{x}\right)+a_{t}
$$

sendo $\bar{x}$ a média da série de observações, $\phi$ são os parâmetros autoregressivos, e at é o ruído branco.

Os modelos autoregressivos com variável exógena ARx podem ser considerados como uma extensão dos modelos AR, sendo que, neste caso, uma nova série temporal também é usada para descrever a variável $\mathrm{x}$, medida num instante de tempo t. Com esse acréscimo, o modelo ARx pode ser escrito na forma da Equação 2. 


$$
x(t)+a_{1} x(t-1)+\ldots+a_{n a} x(t-n a)=b_{0} u(t)+b_{1} u(t-1)+\ldots+b_{n b} u(t-n b)+e(t)(2)
$$

sendo que o índice $t$ representa o passo de tempo das variáveis, $x$ é a variável de resposta, $u$ é a variável exógena, $\mathrm{e}(\mathrm{t})$ corresponde ao ruído, na e nb são as ordens dos polinômios da regressão, e ai e bi são os parâmetros do modelo autoregressivo.

Os parâmetros dos modelos AR e ARx podem ser determinados por diferentes métodos, como o dos mínimos quadrados e o de Yule-Walker.

Para o caso do modelo ARx , foi adotado um único modelo para previsão de vazão com antecedência de três meses, cuja combinação da ordem do modelo, considerando a defasagem das informações de vazão, de precipitação e do fator de sazonalidade, apresentou o menor erro em relação aos valores observados no período de ajuste do modelo. A geração dos cenários de vazão foi feita utilizando os 24 membros da previsão de precipitação por conjunto do modelo ECHAM 4.5, considerando 24 condições iniciais diferentes, no horizonte de um a três meses.

Para o caso da modelagem do tipo AR, foram construídos 24 modelos para gerar os cenários de vazão. Esses 24 modelos foram resultados da combinação de dois diferentes métodos de obtenção dos parâmetros do modelo - o método dos mínimos quadrados e o método de Yule-Walker - e de 12 diferentes defasagens de vazão. Foram, então, feitas 24 previsões de vazão para o horizonte de um a três meses a frente.

O período de calibração e validação dos modelos foi de janeiro de 1950 a dezembro de 2001, sendo 26 anos utilizados para calibração dos modelos e igual período empregado para a correspondente validação. O período de testes correspondeu à geração de cenários de vazão para 6 anos - 2002 a 2007.

\subsection{Métricas para avaliação dos resultados}

Os cenários de vazão gerados foram comparados aos dados observados a partir de diferentes métricas. Essa comparação foi feita entre a vazão observada e a média das 24 previsões do modelo ARx e das 24 previsões dos modelos AR. Em ambos os casos, tanto para os modelos AR quanto para o modelo ARx, também foi tomada a vazão máxima e mínima das 24 previsões, a fim de melhor se avaliar a amplitude dos cenários.

Os índices estatísticos calculados foram: i) erro médio absoluto; ii) erro médio relativo; iii) raiz do erro médio quadrático - REQM; iv) correlação linear de Pearson; v) destreza; vi) coeficiente de eficiência de Nash-Sutcliffe NS; vii) distância multicritério - DM. Maiores detalhes sobre esses índices estatísticos podem ser encontrados em Saturnino Braga et al. (2009) e em Farias (2012). Destaca-se que, para avaliação dos cenários de vazão, a destreza corresponde a um valor percentual que indica melhora da previsão utilizando o modelo ARx quando comparado aos modelos AR.

\section{Resultados}

Os resultados apresentados, nesta seção, correspondem à análise da geração de cenários de afluência para a UHE Furnas, na bacia do Rio Grande, no período de 2002 a 2007, obtidos a partir da implementação dos modelos AR e ARx.

Os cenários gerados para o $1^{\circ}, 2^{\underline{0}}$ e $3^{\circ}$ mês a frente são mostrados nas Figuras 2, 3 e 4, respectivamente. Nessas figuras, as linhas com tons de cor azul são referentes aos cenários gerados pelo modelo ARx, enquanto as de tons de cor vermelho referem-se aos cenários gerados pelos modelos AR. As linhas tracejadas referem-se aos valores máximos e mínimos, sendo que as linhas em tons mais claros correspondem aos valores mínimos, e as linhas em tons mais escuros são correspondentes aos valores máximos. As linhas contínuas azul e vermelha são as médias dos cenários do modelo ARx e dos modelos AR, respectivamente. Já a linha contínua preta, neste caso, corresponde à vazão observada.

Analisando as Figuras 2, 3 e 4, é possível notar que, de maneira geral, a média dos cenários gerados pelo modelo ARx - linha contínua azul - apresenta uma pequena tendência de superestimar a vazão observada - linha contínua preta. Por outro lado, a média dos cenários gerados pelos modelos AR - linha contínua vermelha - tende a subestimar a vazão observada.

Nessas figuras, pode-se, também, perceber o atraso de um mês das previsões de vazão geradas pelos modelos AR, bem como o aumento da abertura dos cenários na medida em que o horizonte da geração de cenários aumenta, o que fica mais evidente nos cenários gerados pelos modelos AR.

Ao avaliar, com maior detalhamento, os gráficos dispostos nas Figuras 2, 3 e 4, pode-se observar a dificuldade dos modelos AR em simular as situações onde ocorre grande variabilidade na vazão, como nos casos de mudança de inclinação das curvas. Os cenários gerados por esses modelos somente indicam tais mudanças, na curva dos gráficos - hidrógrafa, após ter ocorrido a mudança no mês anterior. Em casos específicos, como, por exemplo, nos meses de janeiro e de fevereiro de 2002, 2003 e 2005, os cenários gerados pelos modelos AR não indicaram, dentro de seu limite, a maior vazão no mês de janeiro, enquanto, no mês subsequente - fevereiro, os modelos indicaram as vazões em ascensão, quando, de fato, foi observada uma recessão. Por outro lado, o modelo ARx captura melhor essa variabilidade, por carregar consigo informações adicionais ao comportamento médio e sazonal das vazões.

Para os cenários gerados utilizando os modelos AR, em $77 \%$, $88 \%$ e $86 \%$ dos meses, a vazão observada esteve dentro do intervalo de cenários gerados para o $1^{\circ}$, $2^{\circ}$ e $3^{\circ}$ mês, respectivamente. Alternativamente, para os cenários gerados utilizando o modelo ARx, esses valores percentuais aumentaram para $96 \%, 95 \%$ e $95 \%$, respectivamente. 
Os índices estatísticos para a avaliação dos cenários, calculados, neste estudo, são apresentados na Tabela 1 . Observando os índices válidos para os valores médios - coluna referente à média, tem-se uma melhora considerável dos cenários gerados pelo modelo ARx, quando comparado aos cenários gerados pelos modelos AR, dado que, na maioria dos casos, os erros médio absoluto e relativo, a DM e a REQM diminuem, enquanto o NS e a correlação aumentam. As exceções a esse comportamento foram o aumento do erro médio relativo no segundo mês e no terceiro mês do horizonte de previsão. Além disso, a destreza foi positiva para os valores médios dos três meses do horizonte de previsão, o que destaca a melhoria no desempenho dos cenários médios gerados utilizando as informações de precipitação. Destaca-se que as correlações entre os dados observados e os cená- rios gerados pelo modelo ARx foram sempre superiores às correlações que consideraram os cenários gerados pelos modelos AR. Esse fato pode refletir a habilidade do modelo ARx em capturar melhor a variabilidade das vazões que o modelo AR.

Outro fator a ser evidenciado é que, para o terceiro mês de previsão, todos os índices referentes aos valores mínimos dos cenários gerados pelo modelo ARx apresentaram-se melhores que os valores gerados pelos modelos AR. Na medida em que os cenários gerados pelos modelos AR tiveram a sua variância aumentada, os índices estatísticos refletiram um pior desempenho. Inclusive, no terceiro mês, foi observado o índice NS negativo, o que é uma indicação de que utilizar as previsões desse modelo pode ser, teoricamente, pior do que utilizar o valor médio das observações.

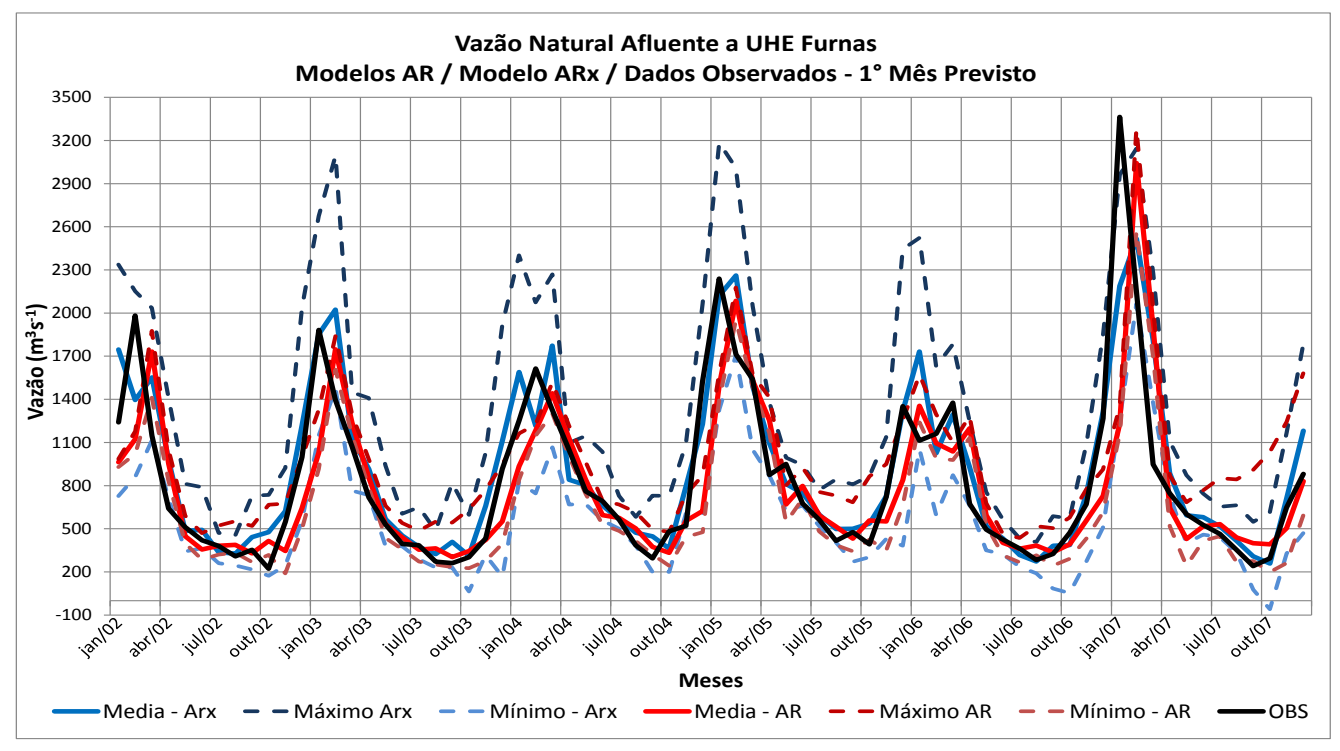

Figura 2 - Cenários de vazão gerados para o $1^{\circ}$ mês

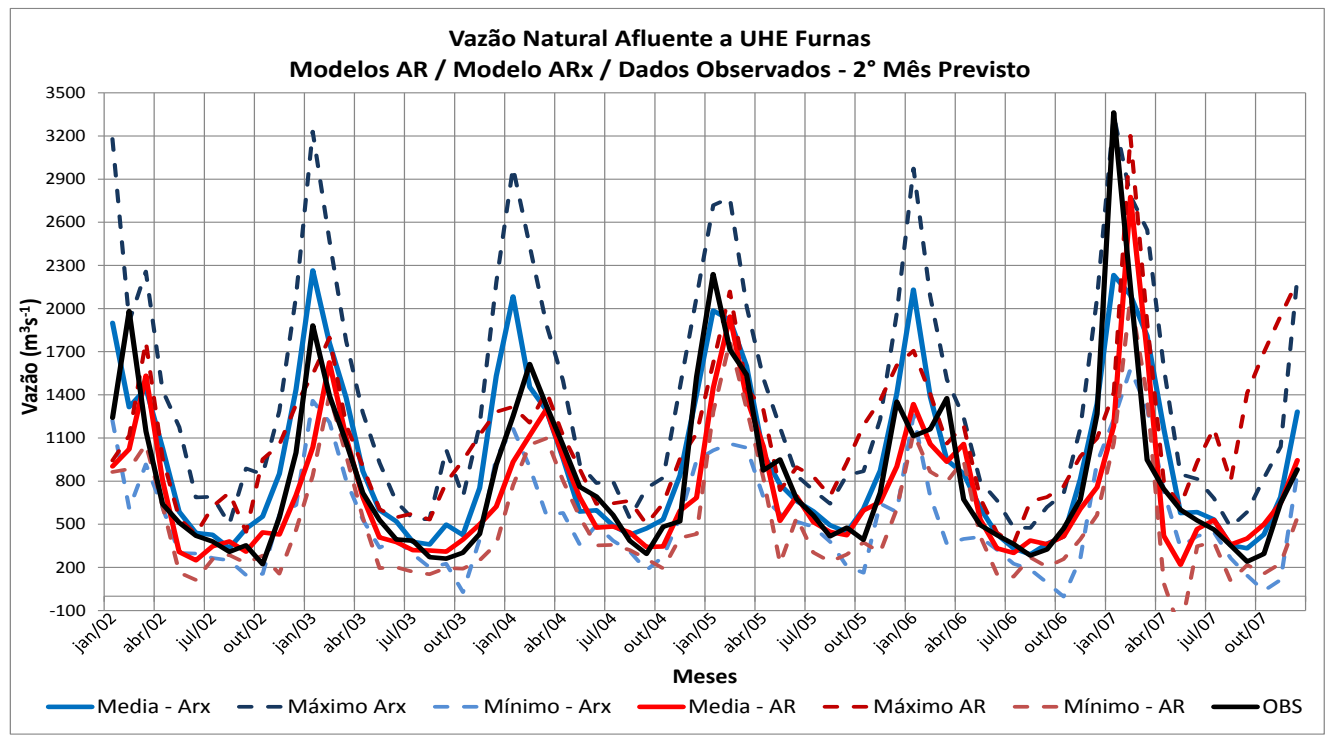

Figura 3 - Cenários de vazão gerados para o $2^{\circ}$ mês 


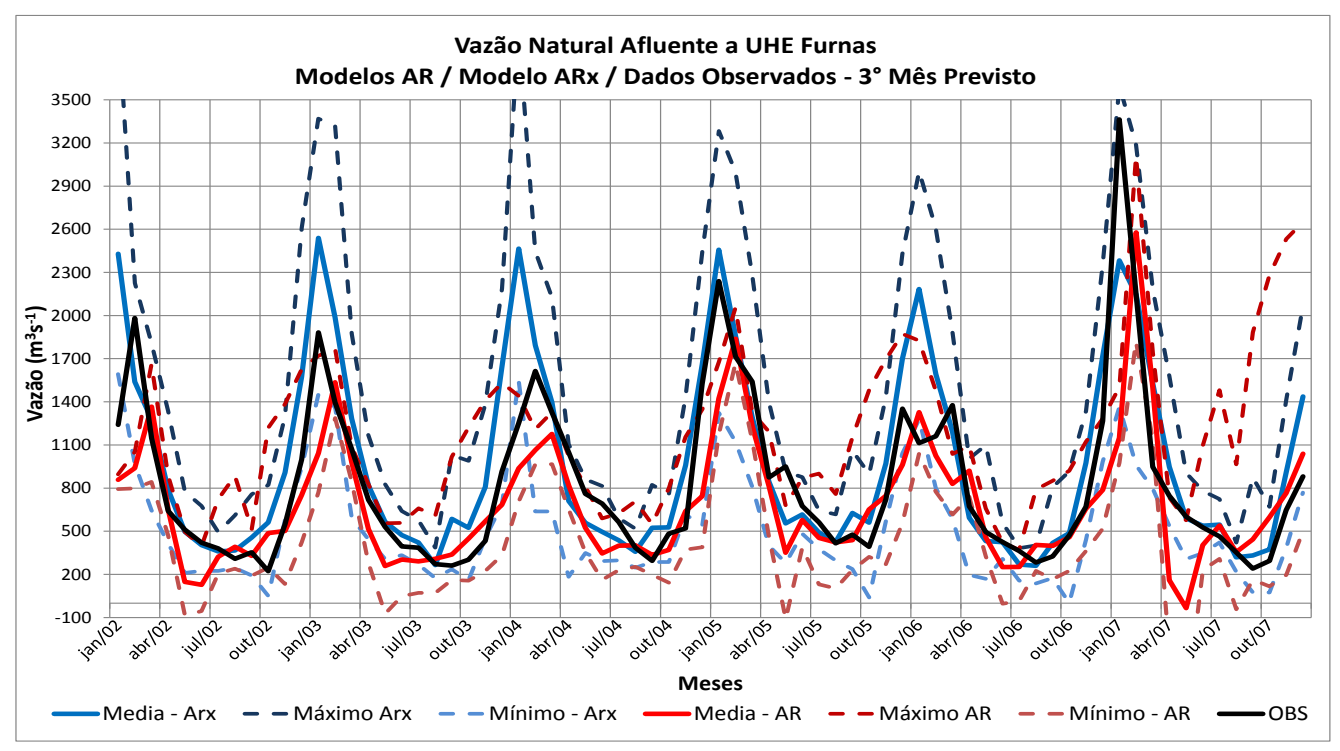

Figura 4 - Cenários de vazão gerados para o $3^{\circ}$ mês

Tabela 1 - Resultados dos índices estatísticos utilizados para avaliação dos cenários de vazão

\begin{tabular}{|c|c|c|c|c|c|c|c|c|c|}
\hline \multirow{2}{*}{ Modelos AR } & \multicolumn{3}{|c|}{ Mês1 } & \multicolumn{3}{|c|}{ Mês 2} & \multicolumn{3}{|c|}{ Mês 3} \\
\hline & Média & Máximo & Mínimo & Média & Máximo & Mínimo & Média & Máximo & Mínimo \\
\hline Erro Médio Absoluto $\left(\mathrm{m}^{3} \mathrm{~s}^{-1}\right)$ & & 328,4 & 261,7 & & 411,5 & 322,5 & & 496,9 & 426,0 \\
\hline Erro Médio Relativo (\%) & & 51,1 & 27,8 & & 73,3 & 39,0 & & 96,2 & 55,4 \\
\hline NS & & 0,21 & 0,28 & & 0,02 & 0,16 & & $-0,10$ & 0,10 \\
\hline DM & & 0,94 & 0,77 & & 1,22 & 0,92 & & 1,47 & 1,06 \\
\hline REQM & & 454,7 & 426,6 & & 552,0 & 479,8 & & 683,9 & 572,6 \\
\hline Correlação & & 0,69 & 0,74 & & 0,59 & 0,76 & & 0,45 & 0,74 \\
\hline \multirow{2}{*}{ Modelo ARx } & \multicolumn{3}{|c|}{ Mês1 } & \multicolumn{3}{|c|}{ Mês 2} & \multicolumn{3}{|c|}{ Mês 3} \\
\hline & \multirow[t]{7}{*}{ Média } & Máximo & Mínimo & Média & Máximo & Mínimo & Média & Máximo & Mínimo \\
\hline Erro Médio Absoluto $\left(\mathrm{m}^{3} \mathrm{~s}^{-1}\right)$ & & 502,8 & 281,0 & & 568,3 & 296,4 & & 655,9 & 337,8 \\
\hline Erro Médio Relativo (\%) & & 71,1 & 32,2 & & 83,7 & 33,9 & & 90,6 & 40,4 \\
\hline NS & & 0,55 & 0,15 & & 0,47 & 0,02 & & 0,44 & 0,11 \\
\hline DM & & 0,84 & 0,91 & & 0,99 & 1,04 & & 1,07 & 0,98 \\
\hline REQM & & 617,3 & 444,9 & & 714,2 & 459,5 & & 872,3 & 473,7 \\
\hline Correlação & & 0,89 & 0,79 & & 0,85 & 0,79 & & 0,83 & 0,79 \\
\hline \multirow{2}{*}{ Destreza(\%) } & Média & Máximo & Mínimo & Média & Máximo & Mínimo & Média & Máximo & Mínimo \\
\hline & & $-35,76$ & $\begin{array}{r}-4,31 \\
\end{array}$ & & $-29,39$ & 4,24 & & $-27,55$ & 17,28 \\
\hline
\end{tabular}

\section{Conclusões}

O principal objetivo deste trabalho foi mostrar que é possível obter um aperfeiçoamento do método de geração de cenários de afluências de vazão no horizonte de até três meses, considerando, além da informação de afluências passadas, previsões climáticas de precipitação, oriundas de MCGA.

Os resultados mostraram que tanto os modelos AR quanto o modelo ARx representaram bem o comportamento sazonal da vazão, no entanto a metodologia univariada apresentou qualidade inferior à multivariada, dado que os modelos $\mathrm{AR}$, de uma maneira geral, não conseguiram antecipar a variação da vazão, tendo dificuldades de capturar a sua variabilidade natural. Os modelos AR exibiram, também, um erro característico da modelagem autoregressiva univariada: os cenários de vazão gerados apresentaram um desvio de fase de um mês em relação à vazão observada, provocando um efeito conhecido como "efeito sombra" nos gráficos dos cenários de vazão, o que foi minimizado com os cenários gerados pelo modelo ARx.

A vazão observada situou-se, na maior parte do período analisado, dentro do intervalo máximo e mínimo dos cenários gerados pelo modelo ARx. O melhor desempenho do modelo ARx, quando comparado aos modelos AR, é evidenciado na análise dos índices estatísticos. A inserção da previsão climática de precipitação por conjuntos mostrou-se como um importante complemento à modelagem estocástica univariada. A incerteza contida 
na previsão por conjuntos de precipitação foi capaz de levar o modelo autoregressivo multivariado a capturar, na grande maioria das vezes, o domínio de soluções possíveis do estado da vazão futura. Com o modelo ARx, os cenários gerados foram capazes de capturar a variabilidade do estado da vazão futura, não ficando a simulação do modelo autoregressivo sujeita, somente, a reproduzir a variabilidade histórica das vazões e o seu comportamento sazonal. É importante destacar que, mesmo com os erros dos modelos climáticos em relação à simulação da variável precipitação, pode-se concluir que a sua utilização em modelos de geração de cenários é promissora, levando-se em consideração, também, que esses MCGA's tendem a possuir melhores resultados no futuro.

Pode-se concluir, com base neste estudo, que a modelagem estocástica univariada pode não ser a mais adequada para capturar a variabilidade de cenários hidrológicos futuros, e que a inclusão de previsões climáticas como variável exógena nessa modelagem pode contribuir de forma significativa para uma melhor representação desses cenários, não só em relação a sua tendência, mas também em relação a sua dispersão.

\section{Referências}

BLOCK, P. J. et al. "A streamflow forecasting framework using multiple climate and hydrological models". Journal of the American Water Resources Association, v.45, n.4, p. $828-843.2009$.

BUIZZA, R.. The ECMWF ensemble prediction system. Relatório técnico do European Centre for Medium-Range Weather Forecasts. 2005.

CATALDI, M. et al. "Assessment of precipitation climate forecasting of models ECHAM 4.5, CFS, COLA/IRI and CCM3 for hydro basins in southwest brazil". In: CLIVAR VAMOS Workshop on Modeling and Predicting Climate in the Americas, Petropolis - RJ. 2012.

CEPEL - CENTRO DE PESQUISA DE ENERGIA ELÉTRICA. Manual de referência - Modelo de geração de séries sintéticas de energias e vazões - MODELO GEVAZP. Rio de Janeiro - RJ. 2002.

CLARKE, R. T. "Hydrological prediction in a nonstationary world". Hydrology and Earth System Sciences, v.11, n.1, p. 408-414. 2007.

DAHER DE DEUS, M.L. Séries temporais aplicadas ao planejamento da operação do Sistema Interligado Nacional - SIN. Dissertação (Mestrado em Engenharia Elétrica). Pontifícia Universidade Católica do Rio de Janeiro, Rio de Janeiro - RJ. 2008.
FARIAS, W. C. M. Aplicação de um modelo autoregressivo multivariado para geração de cenários de afluências incorporando informações climáticas. (Mestrado em Engenharia Civil). Universidade Federal do Rio de Janeiro, Rio de Janeiro - RJ. 2012.

GODDARD, L. et al. "Proveding seasonal -to-interannual climate information for risk management and decisionmaking". Procedia Environmental Sciences, v.1, p.81101. 2010

GUETTER, A. K.,; KRAUSKOPF NETO, R.. “Previsão hidrológica com estimador de estado integrado ao modelo chuva-vazão aplicado a uma bacia da Região Sul". In: Anais do XIV Congresso Brasileiro de Meteorologia, Florianópolis - SC. 2006.

GUILHON, L.G. et al. “Comparação de métodos de previsão de vazões naturais afluentes a aproveitamentos hidroelétricos". Revista Brasileira de Recursos Hídricos, v.12, n.3, p. 13-20. 2007.

HABETS, F. et al. "On the utility of operational precipitation forecasts to served as input for streamflow forecasting". Journal of Hydrology, v.293, p 270 - 288. 2004

PENNA, D.D.J. Definição da árvore de cenários de afluências para o planejamento da operação energética de médio prazo. Tese (Doutorado em Engenharia Elétrica). PontifíciaUniversidade Católica do Rio de Janeiro, Rio de Janeiro - RJ. 2009.

ROECKNER, E. et al. The atmospheric general circulation model ECHAM4: model descripition and simulation of the present-day climate. Max Planck Institut fur Meteorologie, Report n.28, Hamburg, Germany, 90p. 1996.

SANKARASUBRAMANIAN, A. et al. "Role of retrospective of GCMs forced with persisted SST anomalies in operational streamflow forecast development". Journal of Hydrometeorology, v.9, .212-227. 2008.

SATURNINO BRAGA, R. S. et al. "Metodologia para previsão de vazões na bacia do rio Grande". In: Anais do XVIII Simpósio Brasileiro de Recursos Hídricos, Campo Grande-MS. 2009.

SOUZA FILHO, F.A.; LALL, U.. "Seasonal to interannual ensemble streamflow forecasts for Ceara, Brazil: Applications of a multivariate, semiparametric algorithm". Water Resources Research,v.39, n.11, p. 1307. 2003.

WILKS, D.S.. Statistical methods in the atmospheric sciences. Academic Press, 2nd. Edition, 627 p. 2006. 